\title{
Relevancia de la visión crítica de seguridad como paso estandarizado en la colecistectomía laparoscópica
}

\author{
Relevance of the critical view of safety as a standardized step in laparoscopic \\ cholecystectomy
}

\section{Guillermo R. Guevara-Morales*}

Servicio de Cirugía General, Hospital Regional de Veracruz, Instituto de Seguridad y Servicios Sociales de los Trabajadores del Estado, Veracruz, Veracruz, México

A los editores,

Recientemente se publicó en la guía del manejo quirúrgico de la colecistitis aguda, perteneciente a las Guías de Tokio de 2018, una lista estandarizada de pasos de seguridad en la colecistectomía laparoscópica, la cual se basa en el consenso de Delphi, con el objetivo de realizar una colecistectomía laparoscópica segura (Fig. 1). Uno de los pasos que se incluye en esta lista es la creación de la visión crítica de seguridad (VCS), siempre que sea posible; teniendo algunas alternativas de procedimientos de rescate, como la colecistectomía subtotal, en pacientes con situaciones que no permitan realizar la disección del triángulo de Calot para obtener la VCS 1,2 .

La VCS es un método descrito en 1992 y publicado en 1995 por el Dr. Steven M. Strasberg. Posteriormente, en 2013, Sanford y Strasberg propusieron realizar una documentación fotográfica de la VCS con la finalidad de incrementar la seguridad en la colecistectomía laparoscópica. Esta fotodocumentación consiste en calificar mediante un puntaje establecido a la visión anterior y posterior de la VCS, utilizando el término «visión en par» (doublet view). Obtener una VCS satisfactoria, además, forma parte de otros programas de colecistectomía segura, como el implementado por la Society of American Gastrointestinal and Endoscopic Surgeons, entre otros ${ }^{3,4}$. Si bien la VCS constituye un excelente método para la prevención de la lesión de vía biliar, y quizás sea el paso con mayor importancia en la lista que presentan las Guías de Tokio de 2018 para el manejo quirúrgico de la colecistitis aguda, la difusión parcial que ha tenido la VCS en la práctica quirúrgica puede ser una de las principales limitaciones en el desarrollo de la llamada cultura de la colecistectomía segura. En 2017, el Dr. Strasberg señaló que había detectado que, después de 20 años, muchos cirujanos poseen poco entendimiento de los criterios requeridos para lograr la VCS, en especial aquellos que no tuvieron entrenamiento en implementar la VCS en la colecistectomía laparoscópica durante su residencia, por lo que optan por métodos más sencillos, como la técnica infundibular, lo que representa un mayor riesgo de lesión de la vía biliar. Otro de los obstáculos mencionados es que cuando se lleva a cabo la VCS generalmente no se realiza la documentación fotográfica con la calificación de la «visión en par»5.

En conclusión, realizar una colecistectomía laparoscópica mediante pasos de seguridad, con disección, a fin de obtener los tres criterios de la VCS, disminuye el riesgo de un evento adverso, incluida la lesión de la vía biliar. Incluso ante la presencia de alguna complicación seria, que conlleve a un caso médico-legal, la realización de la colecistectomía laparoscópica

\footnotetext{
Correspondencia:

*Guillermo R. Guevara-Morales

Av. Díaz Mirón esquina Sayula, s/n

Fracc. Moderno

C.P. 91918, Veracruz, Ver., México

E-mail: grobertoguevara@gmail.com
}

Fecha de recepción: 19-11-2018

Fecha de aceptación: 15-12-2018

DOI:10.24875/CIRU.18000901
Cir Cir. 2019;87:477-478

Contents available at PubMed www.cirugiaycirujanos.com 

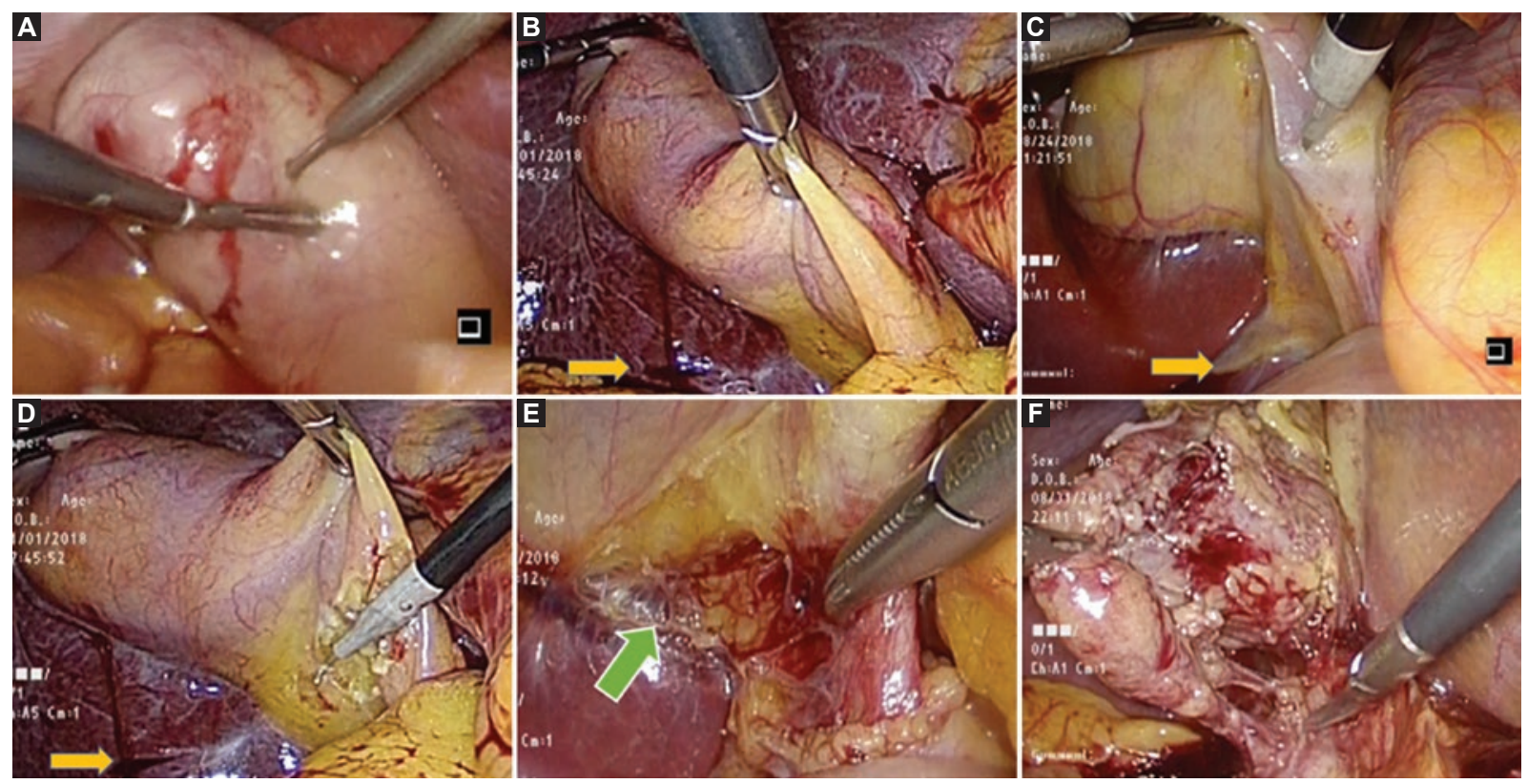

Figura 1. Pasos de seguridad estandarizados en colecistectomía laparoscópica según las Guías de Tokio de 2018. A: Ante una vesícula biliar distendida, que interfiera con el campo de visión, debe ser descomprimida por aspiración mediante una cánula con aguja. B: Realizar tracción efectiva de la vesícula biliar para lograr un plano en el área del triángulo de Calot e identificar sus límites (contratracción). C: Comenzar con la disección en la hoja posterior del peritoneo que cubre el cuello de la vesícula biliar y exponer la superficie de la vesícula biliar por encima del surco de Rouvière. D: Mantener un plano de disección en la superficie de la vesícula biliar durante la colecistectomía laparoscópica. E: Disecar la parte inferior del lecho vesicular (flecha verde) por lo menos un tercio, para obtener una visión crítica de seguridad. F: Realizar la disección de las estructuras para lograr una visión crítica de seguridad. Lograr la hemostasia por compresión y evitar un uso excesivo de electrocauterio o clips. (Las flechas amarillas indican el surco de Rouvière.).

mediante pasos de seguridad estandarizados puede ser la única evidencia que determine de forma contundente si existe o no responsabilidad judicial por una complicación mayor.

\section{Conflicto de intereses}

Los autores declaran no tener conflicto de intereses.

\section{Responsabilidades éticas}

Protección de personas y animales. Los autores declaran que para esta investigación no se han realizado experimentos en seres humanos ni en animales.

Confidencialidad de los datos. Los autores declaran que en este artículo no aparecen datos de pacientes.

\section{Derecho a la privacidad y consentimiento infor-} mado. Los autores han obtenido el consentimiento informado de los pacientes y/o sujetos referidos en el artículo. Este documento obra en poder del autor de correspondencia.

\section{Bibliografía}

1. Iwashita Y, Hibi T, Ohyama T, Umezawa A, Takada T, Strasberg SM, et al. Delphi consensus on bile duct injuries during laparoscopic cholecystectomy: an evolutionary cul-de-sac or the birth pangs of a new technical framework? J Hepatobiliary Pancreat Sci. 2017;24:591-602.

2. Wakabayashi G, Iwashita Y, Hibi T, Takada T, Strasberg SM, Asbun HJ, et al. Tokyo Guidelines 2018: surgical management of acute cholecystitis: safe steps in laparoscopic cholecystectomy for acute cholecystitis. J Hepatobiliary Pancreat Sci. 2018;25:73-86.

3. Sanford DE, Strasberg SM. A simple effective method for generation of a permanent record of the critical view of safety during laparoscopic cholecystectomy by intraoperative "doublet" photography. J Am Coll Surg. 2014;218:170-8.

4. Society of American Gastrointestinal and Endoscopic Surgeons. The SAGES safe cholecystectomy program. 2017. Manages by BSC Management, Inc. Disponible en: https://www.sages.org/safe-cholecystectomy-program

5. Strasberg SM. A perspective on the critical view of safety in laparoscopic cholecystectomy. Ann Laparosc Endosc Surg. 2017;2:91. 